\title{
Research on Obstacle Avoidance Method for Mobile Robot Based on Multisensor Information Fusion
}

\author{
Chengguo Zong, ${ }^{1}$ Zhijian $\mathrm{Ji}^{2 *}$ Yan ${ }^{2}$, ${ }^{1}$ and Hao Shi ${ }^{1}$ \\ ${ }^{1}$ College of Mechanical and Electronic Engineering, Shandong University of Science and Technology, \\ Qingdao, Shandong 266590, China \\ ${ }^{2}$ Institute of Complexity Science, College of Automation, Qingdao University, Qingdao, Shandong 266071, China
}

(Received July 31, 2019; accepted December 9, 2019)

Keywords: mobile robots, obstacle avoidance, multi-sensor information fusion, fuzzy neural network

With the wide application of mobile robots in unstructured environments, an obstacle avoidance system with good performance has become an important part of mobile robot systems. We propose an obstacle avoidance method for a mobile robot based on multi-sensor information fusion technology and a fuzzy neural network control algorithm. In view of complex working environments, a differential kinematics estimation model of a mobile robot is studied. A multi-sensor information fusion method based on the extended Kalman filter and a mobile robot obstacle avoidance algorithm based on fuzzy neural network control are then proposed. Finally, simulations and experiments are conducted, which demonstrate the effectiveness of the proposed method.

\section{Introduction}

Mobile robots are suitable for accomplishing tasks that other robots cannot because they are suited to more complex and varied environments or terrains as a result of their locomotion. To date, mobile robots have been used in many missions such as surveillance in potentially hazardous zones or buildings, dangerous object inspection, searching for survivors in collapsed buildings, gas detection in coal mines, disaster rescue, on the battlefield, and for planetary exploration. Obstacle avoidance ability is a basic need for all mobile robots, which helps them move without collision in unstructured environments. ${ }^{(1-3)}$

During the past few years, obstacle avoidance technology for mobile robots has been investigated by many researchers. ${ }^{(4,5)}$ For example, Yang et al. designed a neural network enhanced telerobot control system and tested it on a Baxter robot. ${ }^{(6)}$ Many robot obstacle avoidance systems with various designs that consist of a collision detection system and sensing modules have been developed. The location of an obstacle can be detected from sensing devices, such as infrared sensors and ultrasonic sensors, and a variety of modules, such as GPS integration and vision systems. ${ }^{(7-10)}$ The data collected from these sensors are the distances between the sensors and an object, which are adjusted and given directly to the control input. ${ }^{(11-13)}$ Therefore, the locations of sonar sensors are very important.

*Corresponding author: e-mail: jizhijian@pku.org.cn https://doi.org/10.18494/SAM.2020.2540 
Multi-sensor information fusion technology means that information will be collected and fused by each sensor to be analyzed, and contradictions between merged information and redundancy will be eliminated to better describe the environment and improve the flexibility and reliability of the system. ${ }^{(14)}$ Since the 1980 s, researchers worldwide have been committed to research based on multi-sensor fusion technology. A single sensing device cannot obtain accurate environmental information, and thus mobile robots based on multi-sensor technology have been developed. More detailed environmental information can be obtained via fused information collected from different types of sensors. At present, there are many fusion methods that can be roughly divided into two categories: probability statistics methods and artificial intelligence methods. ${ }^{(15)}$

Given the specific needs of different mobile robot applications, especially in navigation, the development of an autonomous robotic system that can avoid obstacles while following a path in real-time applications is crucial. Consequently, an efficient collision avoidance and pathfollowing technique is essential to ensure intelligent and effective autonomous mobile robot systems. $^{(16,17)}$

In view of previous research, here, we propose an obstacle avoidance method for a mobile robot. In contrast with the traditional obstacle avoidance methods, ${ }^{(18-20)}$ a fuzzy neural network control algorithm and a sensor detection system consisting of several sensors are proposed. The proposed approach utilizes multi-sensor information fusion method to complete the task and involving a reasonable level of calculations, so that it can be easily used in mobile robot obstacle avoidance. First, a differential kinematics estimation model for a mobile robot that considers complex working environments is presented. Owing to the uncertainty of the mobile robot position, a multi-sensor information fusion method based on the extended Kalman filter (EKF) and a robot obstacle avoidance algorithm based on fuzzy neural network control are proposed. Finally, the effectiveness of the obstacle avoidance technology is verified by simulation and experiment.

This paper is structured as follows: (a) to provide the differential kinematics estimation model of the mobile robot; (b) to develop a multi-sensor information fusion method based on EKF; and (c) to propose the robot obstacle avoidance algorithm based on fuzzy neural network control. Our simulations and experiments demonstrate the effectiveness of the proposed method.

\section{Differential Kinematics Model of Mobile Robot}

As shown in Fig. 1, the differential kinematics estimation model of a mobile robot proposed in this paper adopts the traditional arc model. Let $\left[x_{k}, y_{k}, \alpha_{k}\right]^{\mathrm{T}}$ be the posture of the robot at time $k$; the posture of the robot at time $k+1$ is therefore $\left[x_{k+1}, y_{k+1}, \alpha_{k+1}\right]^{\mathrm{T}}$, where $\left[\Delta x_{k}, \Delta y_{k}, \Delta \alpha_{k}\right]^{\mathrm{T}}$ is the robot posture increment. Let $\Delta s_{l}$ and $\Delta s_{r}$ be the moving distances of the left and right driving wheels of the robot, respectively; the moving distance of the robot during the time interval $(0, \Delta t)$ can be expressed as

$$
\Delta d_{k}=\frac{\Delta s_{l}+\Delta s_{r}}{2}
$$




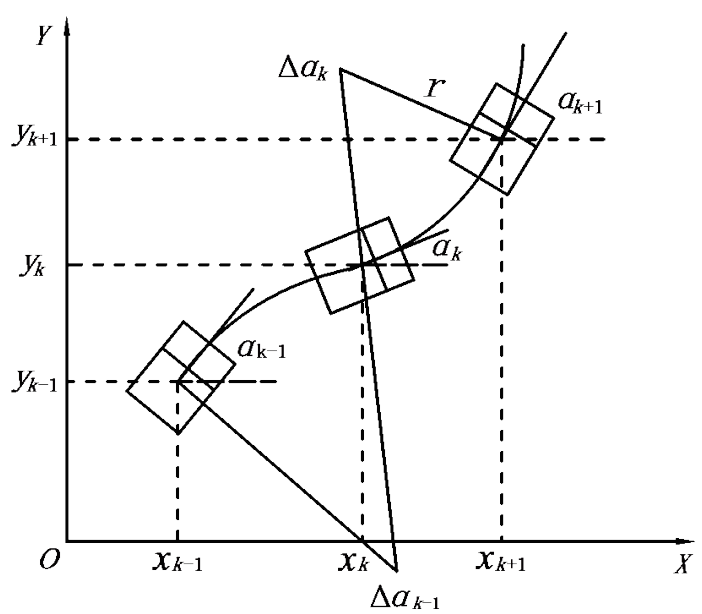

Fig. 1. Differential kinematics model of robot movement.

The rotation angle of the robot during the time interval $(0, \Delta t)$ is

$$
\Delta \alpha_{k}=\frac{\Delta s_{l}+\Delta s_{r}}{B}
$$

where $B$ is the distance between the left and right track wheels of the robot. The rotation radius of the robot is

$$
r=\Delta d_{k} / \Delta \alpha_{k}
$$

The following geometric relationships can be obtained from Fig. 1:

$$
\begin{aligned}
& \Delta x_{k}=2 r \sin \left(\Delta \alpha_{k} / 2\right) \cos \left(\alpha_{k}+\Delta \alpha_{k} / 2\right)=\frac{\Delta d_{k}}{\Delta \alpha_{k}}\left[\sin \left(\alpha_{k}+\Delta \alpha_{k}\right)-\sin \alpha_{k}\right] \\
& \Delta y_{k}=2 r \sin \left(\Delta \alpha_{k} / 2\right) \sin \left(\alpha_{k}+\Delta \alpha_{k} / 2\right)=\frac{\Delta d_{k}}{\Delta \alpha_{k}}\left[\cos \alpha_{k}-\cos \left(\alpha_{k}+\Delta \alpha_{k}\right)\right] .
\end{aligned}
$$

Therefore, the posture of the robot at time $k+1$ can be expressed as

$$
\left\{\begin{array}{c}
x_{k+1}=x_{k}+\Delta x_{k}=x_{k}+\frac{\Delta d_{k}}{\Delta \alpha_{k}}\left[\sin \left(\alpha_{k}+\Delta \alpha_{k}\right)-\sin \alpha_{k}\right] \\
y_{k+1}=y_{k}+\Delta y_{k}=y_{k}+\frac{\Delta d_{k}}{\Delta \alpha_{k}}\left[\cos \alpha_{k}-\cos \left(\alpha_{k}+\Delta \alpha_{k}\right)\right] \\
\alpha_{k+1}=\alpha_{k}+\Delta \alpha_{k}
\end{array} .\right.
$$

Let $U_{k}=\left[\begin{array}{ll}\Delta d_{k} & \Delta \alpha_{k}\end{array}\right]^{\mathrm{T}}$. The state equation of the robot can therefore be expressed as 


$$
X_{k+1}=F\left(X_{k}, U_{k}, W_{k}\right)=\left\{\begin{array}{c}
x_{k}+\frac{\Delta d_{k}}{\Delta \alpha_{k}}\left[\sin \left(\alpha_{k}+\Delta \alpha_{k}\right)-\sin \alpha_{k}\right]+\omega_{1 k} \\
y_{k}+\frac{\Delta d_{k}}{\Delta \alpha_{k}}\left[\cos \alpha_{k}-\cos \left(\alpha_{k}+\Delta \alpha_{k}\right)\right]+\omega_{2 k} \\
\alpha_{k}+\Delta \alpha_{k}+\omega_{3 k}
\end{array} .\right.
$$

When the robot moves in a straight line, Eq. (7) can be simplified to

$$
X_{k+1}=F\left(X_{k}, U_{k}, W_{k}\right)=\left\{\begin{array}{c}
x_{k}+\Delta d_{k} \cos \alpha_{k}+\omega_{1 k} \\
y_{k}+\Delta d_{k} \sin \alpha_{k}+\omega_{2 k}, \\
\alpha_{k}+\omega_{3 k}
\end{array}\right.
$$

where $W_{k}$ is the noise vector expressed as

$$
W_{k}=\left[\begin{array}{lll}
\omega_{1 k} & \omega_{2 k} & \omega_{3 k}
\end{array}\right]^{\mathrm{T}}
$$

Because the dead reckoning algorithm has large errors in the long-distance operation of the robot, it can only be applied to the short-distance positioning of the robot. Therefore, in this paper, the dead reckoning algorithm is only used to predict the posture of the robot in the initial stage.

\section{Multi-sensor Information Fusion Method Based on EKF}

\subsection{EKF model}

Let the state equation of a general system at a certain moment be

$$
X_{k}=F\left(X_{k-1}, U_{k-1}\right)+W_{k-1},
$$

where $X_{k}$ is the state vector of the system and $W_{k}$ is the noise vector.

Its observation equation is

$$
Z_{k}=H X_{k}+V_{k}
$$

where $Z_{k}$ is the observation vector and $V_{k}$ is the observed noise.

The time renewal equation for the EFK is

$$
\left\{\begin{array}{l}
\hat{X}_{k}^{-}=F\left(\hat{X}_{k-1}^{+}, U_{k-1}\right)+W_{k-1} \\
P_{k}^{-}=\nabla F P_{k-1}^{+} \nabla F^{\mathrm{T}}+Q
\end{array}\right.
$$

where $\nabla F$ is the Jacobi matrix of the system. 
The state renewal equation for the EFK is

$$
\left\{\begin{array}{l}
K_{k}=P_{k}^{-} H_{k}^{\mathrm{T}}\left(H_{k} P_{k}^{-} H_{k}^{\mathrm{T}}+r\right)^{-1} \\
\hat{X}_{k}^{+}=\hat{X}_{k}^{-}+K_{k}\left(Z_{k}-\hat{Z}_{k}\right) \\
P_{k}^{+}=\left(I-K_{k} \nabla H\right) P_{k}^{-}
\end{array}\right.
$$

where $\nabla H$ is the Jacobi matrix of the system observation equation for its state variables.

\subsection{Information fusion process of EFK}

The process of estimation of optimal robot posture by using the EFK is as follows. From the estimation of optimal robot posture at the previous moment and the state equation of the system, the current posture of the mobile robot can be estimated. The optimal estimation of the mobile robot posture can be obtained by reasonable updates of the posture estimation. The specific process is illustrated in Fig. 2.

(1) Prediction

According to the posterior estimated pose $X_{k-1}^{+}$of the robot at moment $k-1$ and the derived state equation of the robot, the a priori estimated pose at moment $k$ can be obtained from Eqs. (12) and (13).

The priori posture estimation when the robot moves in a straight line can be expressed as

$$
\hat{X}_{k}^{-}=F\left(\hat{X}_{k-1}^{+}, U_{k-1}, 0\right)=\left[\begin{array}{c}
\hat{X}_{K-1}^{+}+\Delta d_{k-1} \cos \hat{\alpha}_{k-1}^{+} \\
\hat{y}_{K-1}^{+}+\Delta d_{k-1} \sin \hat{\alpha}_{k-1}^{+} \\
\hat{\alpha}_{k-1}^{+}
\end{array}\right] .
$$

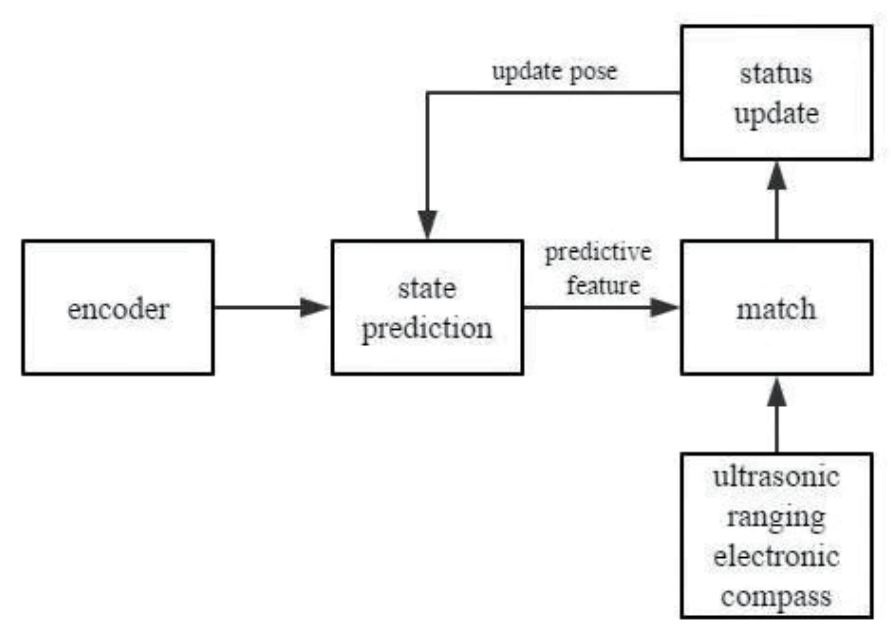

Fig. 2. Process of robotic optimal EKF posture estimation. 
The priori posture estimation when the robot moves in a curved path can be expressed as

$$
\hat{X}_{k}^{-}=F\left(\hat{X}_{k-1}^{+}, U_{k-1}, 0\right)=\left[\begin{array}{c}
\hat{x}_{k-1}^{+}+\frac{\Delta d_{k-1}}{\Delta \alpha_{k-1}}\left[\sin \left(\hat{\alpha}_{k-1}^{+}+\Delta \alpha_{k-1}\right)-\sin \hat{\alpha}_{k-1}^{+}\right] \\
\hat{y}_{k-1}^{+}+\frac{\Delta d_{k-1}}{\Delta \alpha_{k-1}}\left[\cos \hat{\alpha}_{k-1}^{+}-\cos \left(\hat{\alpha}_{k-1}^{+}+\Delta \alpha_{k-1}\right)\right] \\
\hat{\alpha}_{k-1}^{+}+\Delta \alpha_{k-1}
\end{array}\right] .
$$

The covariance is

$$
P_{k}^{-}=\nabla F_{k-1} P_{k-1}^{+} \nabla F_{k-1}^{\mathrm{T}}+Q .
$$

In the case of linear motion,

$$
\nabla F_{k-1}=\left[\begin{array}{ccc}
1 & 0 & -\Delta d_{k-1} \sin \hat{\alpha}_{k-1}^{+} \\
0 & 1 & \Delta d_{k-1} \cos \hat{\alpha}_{k-1}^{+} \\
0 & 0 & 1
\end{array}\right] .
$$

In the case of curved motion,

$$
\nabla F_{k-1}=\frac{\partial F\left(\hat{X}_{k-1}^{+}, U_{k-1}\right)}{\partial \hat{X}_{k-1}^{+}}=\left[\begin{array}{ccc}
1 & 0 & \frac{\Delta d_{k-1}}{\Delta \alpha_{k-1}}\left[\cos \left(\hat{\alpha}_{k-1}^{+}+\Delta \alpha_{k-1}\right)-\cos \hat{\alpha}_{k-1}^{+}\right] \\
0 & 1 & \frac{\Delta d_{k-1}}{\Delta \alpha_{k-1}}\left[\sin \left(\hat{\alpha}_{k-1}^{+}+\Delta \alpha_{k-1}\right)-\sin \hat{\alpha}_{k-1}^{+}\right] \\
0 & 0 & 1
\end{array}\right] .
$$

(2) Observation prediction

The system observations can be expressed as

$$
\hat{Z}_{k}=H_{k} \hat{X}_{k}^{-}=\left[\begin{array}{c}
x_{k-1}^{+}+\frac{\Delta d_{k-1}}{\Delta \alpha_{k-1}}\left[\sin \left(\hat{\alpha}_{k-1}^{+}+\Delta \alpha_{k-1}\right)-\sin \hat{\alpha}_{k-1}^{+}\right] \\
\hat{y}_{k-1}^{+}+\frac{\Delta d_{k-1}}{\Delta \alpha_{k-1}}\left[\cos \hat{\alpha}_{k-1}^{+}-\cos \left(\hat{\alpha}_{k-1}^{+}+\Delta \alpha_{k-1}\right)\right] \\
\hat{\alpha}_{k-1}^{+}+\Delta \alpha_{k-1}
\end{array}\right]
$$

where $H_{k}$ is the system observation matrix. 


\section{(3) Observation}

The robot coordinates based on the observations of the ultrasonic sensors, infrared sensors, and electronic compasses are $Z_{k}=\left[x_{k}, y_{k}, \alpha_{k}\right]^{\mathrm{T}}$.

(4) Match

The difference and covariance matrices between the observed value $Z_{k}$ and predicted value $\hat{Z}_{k}$ can be obtained as

$$
\begin{gathered}
R_{k}=Z_{k}-\hat{Z}_{k} \\
S=E\left(R_{k} R_{k}^{\mathrm{T}}\right)=H_{k} P_{k}^{-} H_{k}^{\mathrm{T}}+r .
\end{gathered}
$$

(5) Update

The Kalman gain of the system can be obtained as

$$
K_{k}=P_{k}^{-} H_{k}^{\mathrm{T}}\left(H_{k} P_{k}^{-} H_{k}^{\mathrm{T}}+r\right)^{-1} .
$$

The robot posture is updated as

$$
\begin{gathered}
\hat{X}_{k}^{+}=\hat{X}_{k}^{-}+K_{k}\left(Z_{k}-\hat{Z}_{k}\right)^{-1} \\
P_{k}^{+}=\left(I-K_{k} H_{k}\right) P_{k}^{-} .
\end{gathered}
$$

\section{Verification of Fuzzy Neural Network Obstacle Avoidance Algorithm}

\subsection{Determination of input variables and membership functions}

In view of the characteristics of strong robustness and independence for an accurate mathematical model, the fuzzy neural network control algorithm is used in robot obstacle avoidance. On the basis of the control requirements of the mobile robot obstacle avoidance system, the number of fuzzy divisions of the distance information input is determined to be 1.5. When the distance measured by the sensor is greater than or equal to $1.5 \mathrm{~m}$, the distance information is marked as $1.5 \mathrm{~m}$ and the system input is "far". At this time, the robot continues to move forward without obstacle avoidance processing. When the measured distance of the sensor is less than $1.5 \mathrm{~m}$, the system input is "near". At this time, the robot turns left or right according to the distance information of the obstacles measured on the left and right sides to smoothly avoid the obstacle.

The fuzzy segmentation number of the target angle input of the obstacle avoidance system controller is determined as 3, i.e., the system fuzzy variable is $[N, Z, P]=[$ the target position is located on the left side of the central axis of the robot body and its angle is negative; the target 
position is on the central axis of the robot body and its angle is zero; the target position is on the right side of the central axis of the robot body and its angle is positive]. The system outputs the velocity membership function of the left and right driving wheels with a value in the range of $[0,1]$, indicating that the velocity range is $0-1 \mathrm{~m} / \mathrm{s}$.

As shown in Fig. 3, which illustrates the system membership functions designed in this paper, $100 \mathrm{~cm}$ is defined as the demarcation between near and far points regarding the distance between the robot body and the obstacle. When the angle between the robot body and the target position is within $\pm 30^{\circ}$, it is 0 , when it is less than $30^{\circ}$, it is negative, and when it is greater than $30^{\circ}$, it is positive; $0.5 \mathrm{~m} / \mathrm{s}$ is defined as the demarcation point between fast and slow for the left and right driving wheels of the robot. The membership functions in Fig. 3 are only used as the initial membership function of the system. After training, they can become the actual membership functions of the fuzzy neural network.

\subsection{Simulation analysis}

The "+" on the left side in Fig. 4 is the starting point of the robot running, the diamond on the right side is the target position of the robot, the circles represent multiple obstacles in the working environment of the robot, and the path composed of "*” symbols represents the track of the robot body. It can be seen from the simulation diagram in Fig. 4(a) that the robot body always keeps a certain distance from the obstacles during the running process and successfully

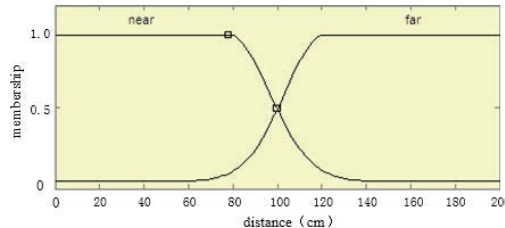

(a)

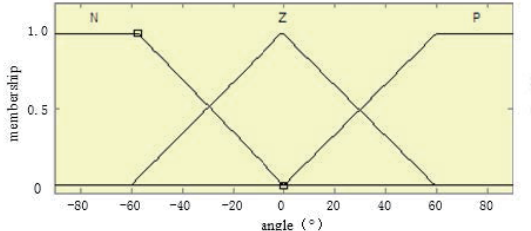

(b)

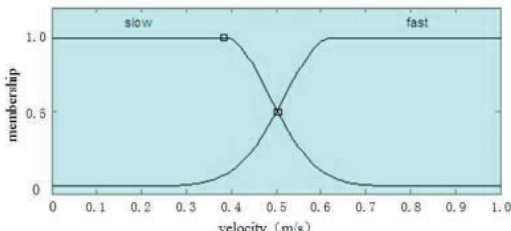

(c)

Fig. 3. (Color online) Membership functions of the (a) distance, (b) angle, and (c) velocity.

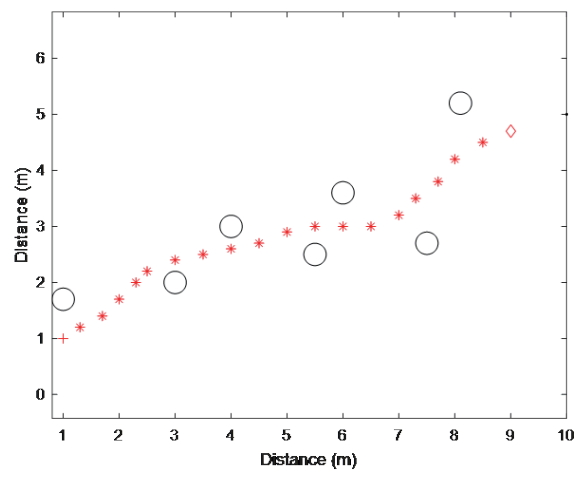

(a)

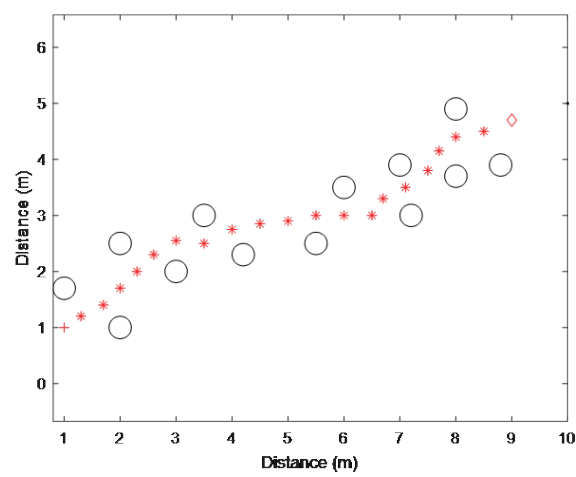

(b)

Fig. 4. (Color online) Schematic diagram of simulations of robot avoidance obstacles. (a) Robot avoidance of obstacles and (b) increasing the number of obstacles. 
reaches the target position. Figure 4(b) presents the simulation results for the obstacle avoidance of the robot after increasing the number of obstacles in the working environment of the robot and changing their positions.

It can be seen from Fig. 4(b) that the robot body still maintains a certain distance from the obstacle during the operation and successfully reaches the target position. It can be concluded that the fuzzy neural network obstacle avoidance control algorithm has high precision and good obstacle avoidance performance.

\section{Robotic Obstacle Avoidance Experiments}

To verify the robot obstacle avoidance technology based on the multi-sensor information fusion method and the fuzzy neural network control algorithm proposed in the previous section, we designed robot obstacle avoidance experiments with a jointed double-track mobile robot platform. The results of obstacle avoidance experiments under two road conditions with the complex robot platform are analyzed and discussed to verify the correctness and feasibility of the theoretical analysis methods proposed in the previous section.

To meet the application requirements of robot obstacle avoidance and a changeable field environment, the multi-sensor detection module adopts a combination of an ultrasonic distance measuring sensor and an infrared sensor. The locations where the sensors are installed are depicted in Fig. 5. The obstacle avoidance system includes a total of 12 sensors, including eight ultrasonic distance measuring sensors and four infrared sensors. The 12 sensors were divided into four groups and installed around the robot body. Each group contained two ultrasonic distance measuring sensors and one infrared sensor. The infrared sensor was installed between two ultrasonic sensors.

From Fig. 6, it is evident that the mobile robot can avoid obstacles smoothly under complicated road conditions and has a good obstacle avoidance function. The correctness of the multi-sensor information fusion method and fuzzy neural network control algorithm proposed in the previous section is verified by the results and analysis of the obstacle avoidance experiments. In addition, the rationality and feasibility of the robot obstacle avoidance method are also clearly illustrated.

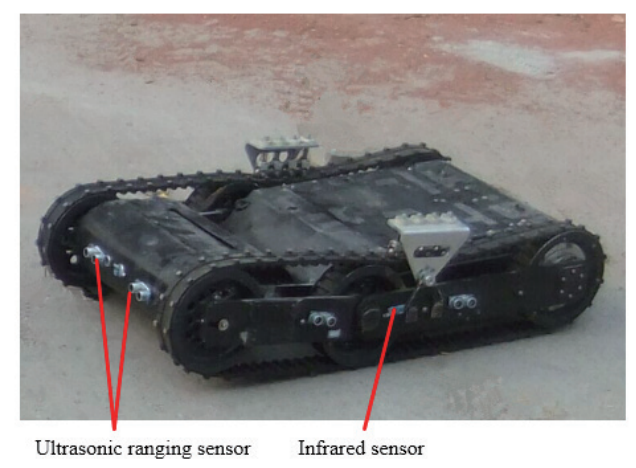

Fig. 5. (Color online) Installation of sensors. 

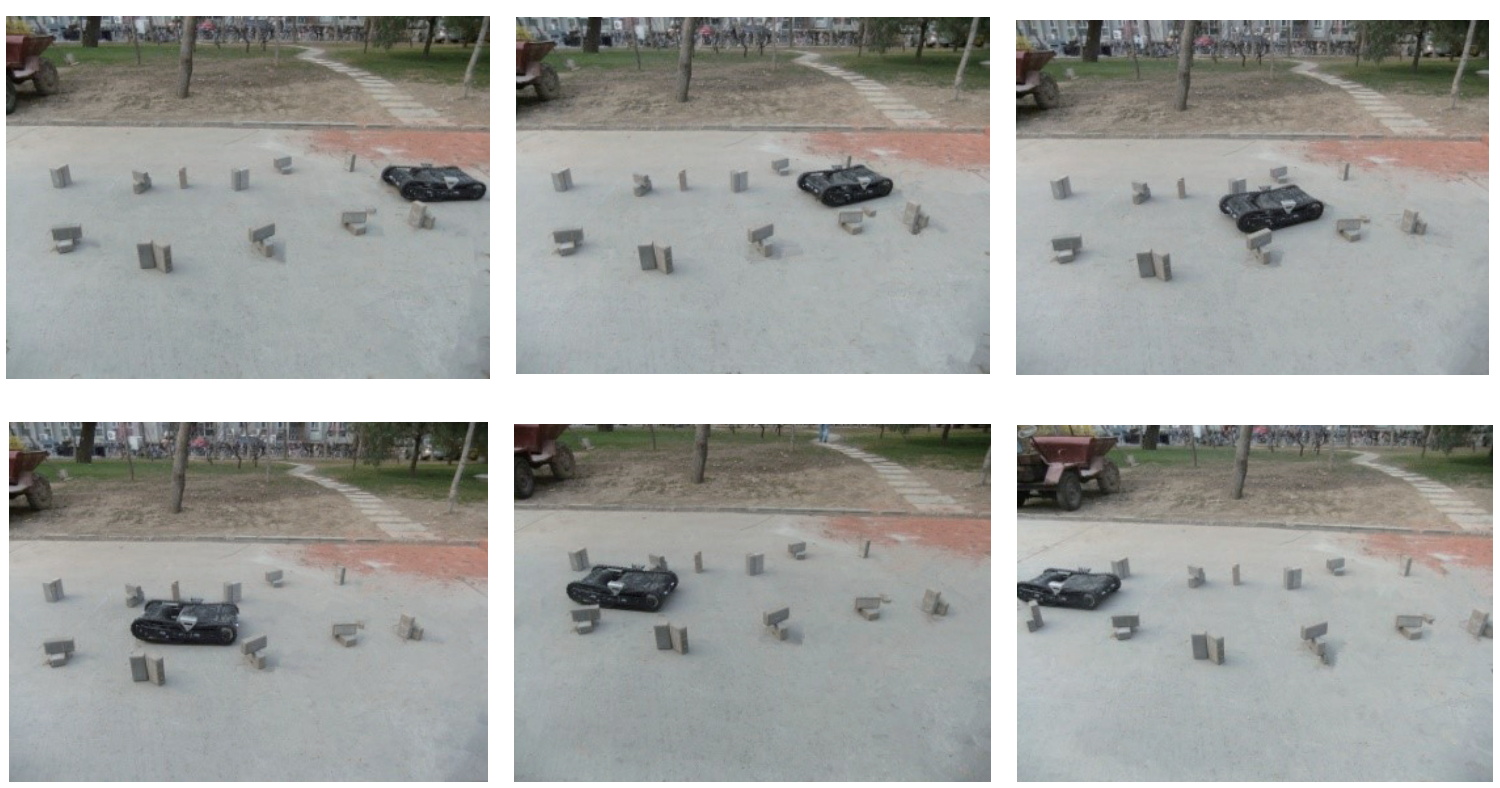

Fig. 6. (Color online) Experiment on robotic obstacle avoidance.

\section{Conclusions}

An obstacle avoidance method for a mobile robot based on multi-sensor information fusion technology and a fuzzy neural network control algorithm was proposed in this paper. Using the traditional arc model, a differential kinematics estimation model of the mobile robot was proposed. The multi-sensor information fusion method based on the EKF and the obstacle avoidance algorithm based on fuzzy neural network control were then applied to mobile robot obstacle avoidance. Simulations and experiments clearly demonstrated the effectiveness of the mobile robot obstacle avoidance method. In future research, the applications of the proposed obstacle avoidance method in multi-robot systems will be studied.

\section{Acknowledgments}

This work was supported by the National Nature Science Foundation of China under Grants 61873136, 61374062, and 61603288 and by the Science Foundation of Shandong Province for Distinguished Young Scholars under Grant JQ201419, Shandong Provincial Natural Science Foundation under Grant ZR201709260010, the Special Funds of Shandong Province for Postdoctoral Innovation Projects under Grant 201702038, and the Postdoctoral Application Research Project of Qingdao.

\section{References}

1 B. Borenstein and Y. Koren: IEEE J. Rob. Autom. 4 (1988) 2. https://doi.org/10.1109/56.2085

2 L. Zeng and G. M. Bone: Int. J. Adv. Rob. Syst. 10 (2013) 2. https://doi.org/10.5772/54933 
3 L. Min, H. R, C. We, and L. Chia: Sens. Mater. 31 (2019) 1. https://doi.org/10.18494/SAM.2019.1993

4 R. E. Tompa, B. Wulfe, M. J. Kochenderfer, and M. P. Owen: J. Aerosp. Inf. Syst. 15 (2018) 2. https://doi. org/10.2514/1.I010576

5 T. Hiroaki. Sens. Mater. 31 (2019) 3. https://doi.org/10.18494/SAM.2019.2155

6 C. Yang, X. Wang, L. Cheng, and Ma H. B. IEEE Tra. Cyb. 47 (2017) 10. https://doi.org/10.1109/ TCYB.2016.2573837

7 A. Mohammed, B. Schmidt, and L. Wang: Int. J. Comput. Integr. Manuf. 30 (2016) 9. https://doi.org/10.1080/0 951192X.2016.1268269

8 W. Retno, A. Tribidasari, S. Endang, and E. Yasuaki: Sens. Mater. 31 (2019) 4. https://doi.org/10.18494/ SAM.2019.2192

9 S. Chen: Sens. Mater. 31 (2019) 5. https://doi.org/10.18494/SAM.2019.2279

10 A. Sarker, C. Qiu, and H. Shen: IEEE Int. Conf. Mobile Ad Hoc \& Sensor Systems (IEEE, 2017) 352-358.

11 P. G. Kim, C. G. Park, Y. H. Jong, and J. H. Yun: Comput. Sci. 15 (2007) 2. https://doi.org/10.1007/978-3-54074171-8_54

12 C. F. Juang and C. H. Hsu: IEEE Trans. Ind. Electron. 56 (2009) 10. https://doi.org/10.1109/TIE.2009.2017557

13 J. O'Keeffe, D. Tarapore, A. G. Millard, and J. Timmis: Conf. Autonomous Robotic Systems (2017) 52-57.

14 H. David and M. Humberto: Sensors 11 (2011) 11. https://doi.org/10.3390/s111110820

15 C. Shao, S. Tanaka, T. Nakayama, and Y. Hata: Sensors 17 (2017) 9. https://doi.org/10.3390/s17091974

16 C. Mineo, S. G. Pierce, P. I. Nicholson, and I. Cooper: J. Comput. Des. Eng. 4 (2017) 3. https://doi.org/10.1016/ j.jcde.2017.01.002

17 W. Wei, J. Huang, and C. Wen: Int. J. Adapt. Control. Signal. Process. 31 (2016) 5. https://doi.org/10.1002/ acs. 2732

18 Y. K. Lee, J. M. Lim, K. S. Eu, and Y. H. Goh: Asia-Pacific Signal \& Information Processing Association Summit \& Conf. (2018) 256-261.

19 X. Li, S. Song, and G. Yong: Nonlinear. Dyn. 93 (2018) 2. https://doi.org/10.1007/s11071-018-4202-5

20 Y. Yang and B. Ding: Circuits Syst. Signal. Process. 38 (2019) 7. https://doi.org/10.1007/s00034-018-1003-5

\section{About the Authors}

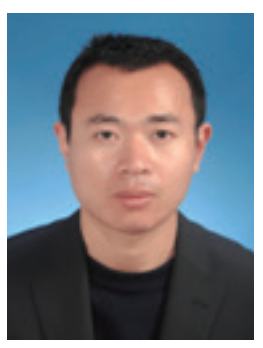

Chengguo Zong (M'83) received his M.S. degree from the College of Mechanical and Electronic Engineering, Shandong University of Science and Technology, China, in 2011 and his Ph.D. degree in mechanical engineering from Beijing Institute University, China, in 2015. Since 2017, he has been engaged in postdoctoral research at the College of Automation of Qingdao University, China. He is currently an assistant professor at Shandong University of Science and Technology. His research interests include mobile robots, industrial robots, new robotic mechanisms, and the formation control of multi-robot systems. (superzcg@163.com)

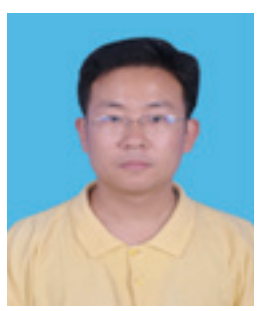

Zhijian Ji (M'73) received his M.S. degree in applied mathematics from Ocean University of China in 1998 and his Ph.D. degree in system theory from the Intelligent Control Laboratory, Center for Systems and Control, Department of Mechanics and Engineering Science, Peking University, China in 2005. He is currently a professor at the College of Automation Engineering, Qingdao University. His current research interests are in the fields of nonlinear control systems, coordination of multi-agent systems, switched and hybrid systems, and formation control and swarm dynamics.

(jizhijian@pku.org.cn) 


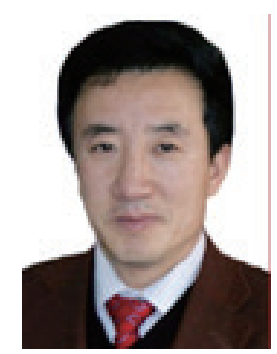

Yan Yu (M'60) received his masters degree in engineering from Shandong University of Science and Technology in 1986. He is currently a professor at Shandong University of Science and Technology. His current research interests are mechatronics, dynamics, and electro-hydraulic integrated control. (yuntisuo@yahoo.com.cn)

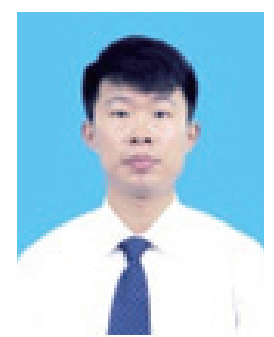

Hao Shi (M'89) received his masters degree in engineering from Shandong University of Science and Technology, China, in 2015 and his Ph.D. degree from Shandong University of Science and Technology in 2018. He is currenty an assistant professor at Shandong University of Science and Technology. His research interests include mechtronics, dynamics, and finite element analysis. (shihao@sdust.edu.cn) 